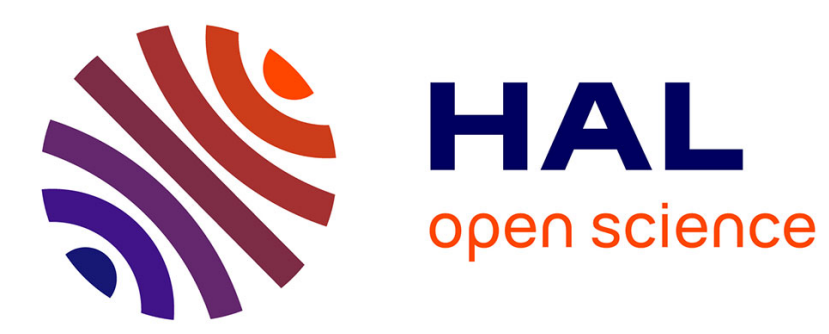

\title{
Time-splitting approximation of the Cauchy problem for a stochastic conservation law \\ Caroline Bauzet
}

\section{To cite this version:}

Caroline Bauzet. Time-splitting approximation of the Cauchy problem for a stochastic conservation law. Mathematics and Computers in Simulation, 2015, 118, pp.73-86. 10.1016/j.matcom.2014.11.012 . hal-01309580

\section{HAL Id: hal-01309580 \\ https://hal.science/hal-01309580}

Submitted on 17 May 2016

HAL is a multi-disciplinary open access archive for the deposit and dissemination of scientific research documents, whether they are published or not. The documents may come from teaching and research institutions in France or abroad, or from public or private research centers.
L'archive ouverte pluridisciplinaire HAL, est destinée au dépôt et à la diffusion de documents scientifiques de niveau recherche, publiés ou non, émanant des établissements d'enseignement et de recherche français ou étrangers, des laboratoires publics ou privés. 


\title{
Time-splitting approximation of the Cauchy problem for a stochastic conservation law
}

\author{
Caroline Bauzet \\ Aix-Marseille Université \\ Institut de Mathématiques de Marseille \\ UMR 7373 \\ 13453 Marseille, France
}

\begin{abstract}
In this paper, we present a time discretization of a first-order hyperbolic equation of nonlinear type set in $\mathbb{R}^{d}$ and perturbed by a multiplicative noise. Using an operator splitting method, we are able to show the existence of an approximate solution. Thanks to recent techniques of well-posedness theory on this kind of stochastic equations, we show the convergence of such an approximate solution towards the unique stochastic entropy solution of the problem, as the time step of the splitting scheme converges to zero.
\end{abstract}

Keywords: Stochastic PDE, multiplicative noise, Itô integral, time-splitting method, Young measures, entropy solution

2014 MSC: 35L60, 60H15, 35L60

\section{Introduction}

We are interested in the Cauchy problem for a nonlinear hyperbolic scalar conservation law with a multiplicative stochastic perturbation of type:

$$
\left\{\begin{aligned}
d u+\operatorname{div} \overrightarrow{\mathbf{f}}(u) d t & =h(u) d W & & \text { in }] 0, T\left[\times \mathbb{R}^{d} \times \Omega,\right. \\
u(\omega, 0, x) & =u_{0}(x), & & \omega \in \Omega, x \in \mathbb{R}^{d},
\end{aligned}\right.
$$

Email address: caroline.bauzet@univ-amu.fr (Caroline Bauzet)

URL: http://www.cmi.univ-mrs.fr/ ${ }^{\sim}$ caroline.bauzet/ (Caroline Bauzet)

Preprint submitted to Journal of Mathematics and Computers in SimulationDecember 8, 2014 
where div is the divergence operator with respect to the space variable (which belongs to $\left.\mathbb{R}^{d}\right), d \geqslant 1, T>0$ and $W=\left\{W_{t}, \mathcal{F}_{t} ; 0 \leq t \leq T\right\}$ is a standard adapted one-dimensional continuous Brownian motion defined on the classical Wiener space $(\Omega, \mathcal{F}, P)$. By denoting $Q=] 0, T\left[\times \mathbb{R}^{d}\right.$, this equation has to be understood in the following way: P-a.s. in $\Omega$ and $\forall \varphi \in \mathcal{D}(Q)$

$$
\int_{Q} u \partial_{t} \varphi+\overrightarrow{\mathbf{f}}(u) \cdot \nabla_{x} \varphi d x d t=\int_{Q} \int_{0}^{t} h(u) d W(s) \partial_{t} \varphi d x d t .
$$

Note that, even in the deterministic case, a weak solution to a nonlinear scalar 5 conservation law is not unique in general. The mathematical stake consists in introducing a selective criterion in order to identify the physical solution. In the present work we consider a stochastic version of the entropy condition proposed by S.N. Kruzhkov in the 70s, the one used in [2] and presented in Section 2 . We assume the following hypotheses:

10 $\quad \mathrm{H}_{1}: \overrightarrow{\mathbf{f}}: \mathbb{R} \rightarrow \mathbb{R}^{d}$ is a Lipschitz-continuous function with $\overrightarrow{\mathbf{f}}(0)=\mathbf{0}$.

$\mathrm{H}_{2}: h: \mathbb{R} \rightarrow \mathbb{R}$ is a Lipschitz-continuous function with $h(0)=0$.

$\mathrm{H}_{3}: u_{0} \in L^{2}\left(\mathbb{R}^{d}\right)$.

$\mathrm{H}_{4}$ : There exists $M>0$ such that $\operatorname{supp} h \subset[-M, M]$.

$\mathrm{H}_{5}: u_{0} \in L^{\infty}\left(\mathbb{R}^{d}\right) \cap B V\left(\mathbb{R}^{d}\right)^{1}$.

\section{Remark 1.}

\footnotetext{
${ }^{1}$ where $B V\left(\mathbb{R}^{d}\right)$ denotes the set of integrable functions with bounded variation on $\mathbb{R}^{d}$.
} 
- $H_{1}, H_{2}$ and $H_{3}$ are claimed conditions from the theoretical point of view to ensure the well-posedness in the sense of [2]. Let us first mention that $H_{1}$ can be weakened by assuming that $\overrightarrow{\mathbf{f}}$ is a locally Lipschitz continuous function. Indeed, since the solution $u$ is bounded by a constant $M_{1}$ depending only on $M$ and $\left\|u_{0}\right\|_{\infty}$, the result holds by a truncation argument of $\overrightarrow{\mathbf{f}}$ outside $\left[-M_{1}, M_{1}\right]$. Secondly, since $\operatorname{div}[\overrightarrow{\mathbf{f}}(0)]=0$, one can assume by convenience that $\overrightarrow{\mathbf{f}}(0)=\mathbf{0}$.

- $H_{4}$ and $H_{5}$ are specific conditions from the numerical analysis point of view. These are technical assumptions to control the estimates in the forthcoming lemmas, in particular to apply Lemma 3.3. Note that $H_{4}$ is a necessary condition to keep the solution u bounded.

\subsection{Former results}

Only few papers have been devoted to the theoretical study of hyperbolic scalar conservation laws with a multiplicative stochastic forcing: the development of a well-posedness theory has been done in [4-[9] by the way of strong entropy solution, in [6] by the use of kinetic formulation, and in [2]-3] with the notion of stochastic entropy solution. For a thorough exposition of all these papers, we refer the reader to the introduction of [1]. Concerning the numerical 35 analysis of such stochastic problems, there is also, to our knowledge, few papers. Let us cite the work of Holden-Risebro [10] where a time-discretization of the 
equation is proposed by the use of an operator-splitting method. They proposed a result of convergence to prove the existence of pathwise weak solutions to the Cauchy problem for (1) set in $\mathbb{R}$. In the recent paper of Bauzet [1, a generalization of the work of Holden-Risebro [10] is proposed in a bounded domain $D$ of $\mathbb{R}^{d}$. The author proved that the pathwise weak solution obtained in [10] is the unique entropy weak solution of the stochastic conservation law and that the whole sequence of approximation given by the time-splitting scheme converges strongly. Kröker-Rohde [11] are interested in a recent work in a method of 45 handling the finite volume schemes for the approximate solution of the Cauchy problem for (1) and investigate on a space-discretization of the equation. For a class of strongly monotone numerical fluxes they established the pathwise convergence of a semi-discrete finite volume solution towards a stochastic entropy solution. Since the authors use a stochastic version of the compensated compactness approach, the study is restricted to the one-dimensional case.

\subsection{Goal of the study and main result}

In a recent published paper [1], a generalization of the time-splitting method introduced much earlier by Holden and Risebro [10] is proposed to approximate solution of the stochastic conservation law (1) set in a bounded domain $D$ of

${ }_{55} \mathbb{R}^{d}$ and with homogeneous Dirichlet boundary condition. Precisely, the author 
showed that the pathwise weak limit obtained in the former study of Holden and Risebro is the unique stochastic entropy solution of (1) and that the whole sequence of approximate solutions converges strongly with respect to all its variables. Our aim in the present paper is to prove that the tools developed 6o in [1] are sufficiently strong to be extended to an unbounded domain and allow us to complete the work of Bauzet-Vallet-Wittbold [2] by a numerical analysis using their well-posedness theory for stochastic entropy solution. The main result of the present paper which deals with the convergence of our numerical scheme is stated in the following theorem.

${ }_{65}$ Theorem 1.1. Assume that hypotheses $H_{1}$ to $H_{5}$ hold. Set $N \in \mathbb{N}^{*}$ and let $\Delta=$ $\frac{T}{N}$ be the time step of the time-spliting operator scheme. Then, the associated approximate solution denoted $u^{\Delta}$ in the sequel and defined p.13 converges in $L_{l o c}^{p}(\Omega \times Q)$ for any finite $p$ towards the unique stochastic entropy solution of the stochastic conservation law (1).

7o The paper is organized as follows. Section 2 is devoted to the presentation of the theoretical background : we recall the definition of a solution for 11 proposed in [2] and their well-posedness result. In Section 3, we present firstly the time-splitting scheme used to approximate the solution of our problem. Several preliminaries results satisfied by the time-splitting approximate solution $u^{\Delta}$ are 
75 then stated. The remainder of Section 3 is devoted to show the convergence of $u^{\Delta}$ towards the unique stochastic entropy solution of 11 . To illustrate our proposal, we present in Section 4 numerical experiments around the stochastic burgers' equation in the one-dimensional case.

\subsection{Notations}

so.$E$ denotes the integral over $\Omega$ with respect to the probability measure $P$.

- Consider $B V\left(\mathbb{R}^{d}\right)$ the set of integrable functions with bounded variation on $\mathbb{R}^{d}$ endowed with the norm $\|v\|_{B V\left(\mathbb{R}^{d}\right)}=\|v\|_{L^{1}\left(\mathbb{R}^{d}\right)}+T V_{x}(v)$, where $T V_{x}(v)$ denotes the total variation of $v$ on $\mathbb{R}^{d}$ (see Evans-Gariepy [7]).

- Denote by $N_{w}^{2}\left(0, T, L^{2}\left(\mathbb{R}^{d}\right)\right)$ the set of the predictable processes of $L^{2}(] 0, T\left[\times \Omega, L^{2}\left(\mathbb{R}^{d}\right)\right)($ Da Prato-Zabczyk[5] p.94).

- Consider $\mathcal{E}$ the set of any $C^{2,1}(\mathbb{R})$ nonnegative convex approximation of the absolute-value function such that $\eta(0)=0$ and that there exists $\delta>0$ such that $\eta^{\prime}(x)=1($ resp. -1$)$ if $x>\delta($ resp. $x<\delta)$.

- $F^{\eta}$ denotes the entropy flux defined for any $a, b \in \mathbb{R}$ and for any smooth entropy $\eta \in \mathcal{E}$ by $F^{\eta}(a, b)=\int_{b}^{a} \eta^{\prime}(\sigma-b) f^{\prime}(\sigma) \mathrm{d} \sigma$. Note in particular that $F^{\eta}$ is a Lipschitz-continuous function. 


\section{Existence and uniqueness result}

Let us recall the definitions and the result introduced in the paper of BauzetVallet-Wittbold [2]. These results are obtained under hypotheses $H_{1}$ to $H_{3}$.

\section{Definition 2.1. (Stochastic entropy solution)}

A function $u$ of $\mathcal{N}_{w}^{2}\left(0, T, L^{2}\left(\mathbb{R}^{d}\right)\right) \cap L^{\infty}\left(0, T ; L^{2}\left(\Omega, L^{2}\left(\mathbb{R}^{d}\right)\right)\right)$ is an entropy solution of the stochastic scalar conservation law (1) with the initial condition $u_{0} \in L^{2}\left(\mathbb{R}^{d}\right)$, if P-a.s in $\Omega$, for any $\eta \in \mathcal{E}$ and for any $(\lambda, \varphi) \in \mathbb{R} \times \mathcal{D}^{+}\left(\left[0, T\left[\times \mathbb{R}^{d}\right)\right.\right.$

$$
\begin{aligned}
0 \leqslant & \int_{\mathbb{R}^{d}} \eta\left(u_{0}-\lambda\right) \varphi(0, x) d x+\int_{Q} \eta(u-\lambda) \partial_{t} \varphi d x d t+\int_{Q} F^{\eta}(u, \lambda) \cdot \nabla_{x} \varphi d x d t \\
& +\int_{0}^{T} \int_{\mathbb{R}^{d}} \eta^{\prime}(u-\lambda) h(u) \varphi d x d W(t)+\frac{1}{2} \int_{Q} h^{2}(u) \eta^{\prime \prime}(u-\lambda) \varphi d x d t .
\end{aligned}
$$

For technical reasons, as in [2], we also need to consider a generalized notion of

\section{Definition 2.2. (Measure-valued entropy solution)}

A function $\mathbf{u}$ of $\mathcal{N}_{w}^{2}\left(0, T, L^{2}\left(\mathbb{R}^{d} \times(0,1)\right)\right) \cap L^{\infty}\left(0, T ; L^{2}\left(\Omega \times \mathbb{R}^{d} \times(0,1)\right)\right)$ is a measure-valued entropy solution of the stochastic scalar conservation law (1) 
with the initial condition $u_{0} \in L^{2}\left(\mathbb{R}^{d}\right)$, if $P$-a.s in $\Omega$, for any $\eta \in \mathcal{E}$ and for any $(\lambda, \varphi) \in \mathbb{R} \times \mathcal{D}^{+}\left(\left[0, T\left[\times \mathbb{R}^{d}\right)\right.\right.$

$$
\begin{aligned}
0 \leqslant & \int_{\mathbb{R}^{d}} \eta\left(u_{0}-\lambda\right) \varphi(0, x) d x+\int_{Q} \int_{0}^{1} \eta(\mathbf{u}(., \alpha)-\lambda) \partial_{t} \varphi d \alpha d x d t \\
& +\int_{Q} \int_{0}^{1} F^{\eta}(\mathbf{u}(., \alpha), \lambda) . \nabla_{x} \varphi d \alpha d x d t \\
& +\int_{0}^{T} \int_{\mathbb{R}^{d}} \int_{0}^{1} \eta^{\prime}(\mathbf{u}(., \alpha)-\lambda) h(\mathbf{u}(., \alpha)) \varphi d \alpha d x d W(t) \\
& +\frac{1}{2} \int_{Q} \int_{0}^{1} h^{2}(\mathbf{u}(., \alpha)) \eta^{\prime \prime}(\mathbf{u}(., \alpha)-\lambda) \varphi d \alpha d x d t .
\end{aligned}
$$

\subsection{Introduction}

Our aim is to approximate Problem (1) under Assumptions $\mathrm{H}_{1}$ to $\mathrm{H}_{5}$. We introduce the method proposed by Holden-Risebro in [10], which consists in splitting the effect of the source term. This technique allows us to construct a$$
\text { on considering the equation in two parts, solving first a stochastic differential }
$$ 
equation, and then using the obtained solution as an initial condition for a homogeneous hyperbolic scalar conservation law. As an extension of [10], we propose in this paper to generalize their estimates on the approximate sequence

125 to the $\mathbb{R}^{d}$-case, in the idea of Chen-Ding-Karlsen [4] concerning BV estimates. Following the notations introduced in [10] we define here two operators for $s$, $t \in[0, T]$ and associated results. Let $R(t, s)$ be the operator which takes a number $\bar{u}$ to the solution $u$ at time $t$ of the stochastic differential equation, $\forall t \in[s, T]$

$$
\left\{\begin{aligned}
d u(t) & =h(u) d W(t) \\
u(t=s) & =\bar{u}
\end{aligned}\right.
$$

130 i.e $u(t)=R(t, s) \bar{u}=\bar{u}+\int_{s}^{t} h(u) d W$.

And $S(t-s)$ denotes the operator which takes an initial function $u(x, s)$ at time $s$ to the weak entropy solution $u$ at time $t$ of the first-order hyperbolic equation

$$
\left\{\begin{array}{rlr}
u_{t}+\operatorname{div} \overrightarrow{\mathbf{f}}(u) & =0 & \text { in }] 0, T\left[\times \mathbb{R}^{d},\right. \\
u(t=s) & =u(x, s), &
\end{array}\right.
$$

i.e $u(x, t)=S(t-s) u(x, s)$.

Lemma 3.1. Consider $s \in[0, T]$. Then P-a.s in $\Omega$ and for all $t \in[s, T]$, ${ }_{135} R(t, s)$ will take $[-M, M]$ into itself and be the identity outside this interval, where $M>0$ is defined in $H_{4}$. 
Proof. Consider the process $u$ defined for all $t \in[s, T]$ by $u(t)=R(t, s) u(s)$. Applying the Itô formula to a regular function $\Psi$ independent of the time variable $t$, vanishing in $[-M, M]$ and increasing outside this interval, one gets, $\mathrm{P}$-a.s:

$$
\begin{aligned}
\Psi(u(t))= & \Psi(u(s))+\int_{s}^{t} \underbrace{\Psi_{t}(u(\sigma))}_{=0} d \sigma+\int_{s}^{t} \underbrace{\Psi_{x}(u(\sigma)) h(u(\sigma))}_{=0} d W(\sigma) \\
& +\frac{1}{2} \int_{s}^{t} \underbrace{\Psi_{x x}(u(\sigma)) h^{2}(u(\sigma))}_{=0} d \sigma, \quad \forall t \in[s, T] .
\end{aligned}
$$

${ }_{140}$ Consider $\omega \in \tilde{\Omega}$, where $\tilde{\Omega}$ is a full measure subset of $\Omega$ and $t \in[s, T]$. Thus, if $u(s, \omega) \in[-M, M], \Psi(u(s, \omega))=0=\Psi(u(t, \omega))$ and $u(t, \omega) \in[-M, M]$. Else, $\Psi(u(t, \omega))=\Psi(u(s, \omega))$, by injectivity of $\Psi$ in $\mathbb{R}-[-M, M], u(t, \omega)=u(s, \omega)$ and $R(t, s)=I_{d}$.

Lemma 3.2. Consider $s \in[0, T], v_{0} \in L^{1}\left(\Omega \times \mathbb{R}^{d}\right) \cap L^{2}\left(\Omega \times \mathbb{R}^{d}\right)$ a $\mathcal{F}_{\text {s-measurable }}$ process such that

$$
E\left[T V_{x}\left(v_{0}\right)\right]<\infty
$$

Define the process $v$ for all $t \in[s, T]$ by $v(t)=R(s, t) v_{0}$. Then for all $t \in[s, T]$

$$
E\left[T V_{x}(v(t))\right] \leqslant E\left[T V_{x}\left(v_{0}\right)\right]
$$

Remark 2. Let us mention that using the lower semi-continuity property and the positivity of the total variation $T V_{x}$ on $L^{1}\left(\mathbb{R}^{d}\right)$, for all $v$ in $L^{1}\left(\Omega \times \mathbb{R}^{d}\right)$, $E\left[T V_{x}(v)\right]$ has a sense.

Proof. Consider $s \in[0, T]$ and let $v_{0} \in L^{1}\left(\Omega \times \mathbb{R}^{d}\right) \cap L^{2}\left(\Omega \times \mathbb{R}^{d}\right)$ be a $\mathcal{F}_{s^{-}}$ measurable process with $E\left[T V_{x}\left(v_{0}\right)\right]<\infty$. Define for all $t \in[s, T] v(t)=$ 
$R(s, t) v_{0}$ and consider $\eta_{\delta} \in \mathcal{E}$. Applying Itô's formula with the process $v$ and the function $\eta_{\delta}$, one gets by taking the integral over $\mathbb{R}^{d}$ and the expectation, for every $t \in[s, T]$

$$
E \int_{\mathbb{R}^{d}} \eta_{\delta}(v(t)) d x=E \int_{\mathbb{R}^{d}} \eta_{\delta}\left(v_{0}\right) d x+\frac{1}{2} E \int_{\mathbb{R}^{d}} \int_{s}^{t} \eta_{\delta}^{\prime \prime}(v(t)) h^{2}(v) d \sigma d x .
$$

155

Passing to the limit on $\delta$ to 0 to get for every $t \in[s, T]$

$$
E\|v(t)\|_{L^{1}\left(\mathbb{R}^{d}\right)}=E\left\|v_{0}\right\|_{L^{1}\left(\mathbb{R}^{d}\right)}
$$

Following the idea of Chen-Ding-Karlsen [4] we consider $v_{0}^{\epsilon}$ a smooth approximation of $v_{0}$ such that

$$
\begin{aligned}
v_{0}^{\epsilon} \rightarrow v_{0} & \text { in } L^{p}\left(\Omega \times \mathbb{R}^{d}\right), p=1,2 \\
E \int_{\mathbb{R}^{d}}\left|\nabla v_{0}^{\epsilon}\right|^{2} d x<\infty, & \text { for each fixed } \epsilon \\
E\left[T V_{x}\left(v_{0}^{\epsilon}\right)\right] \leqslant & E\left[T V_{x}\left(v_{0}\right)\right] .
\end{aligned}
$$

The proof of these results relies on approximation of BV-function in the deterministic setting, we refer the reader to Evans-Gariepy [7] p.172.

Let us define for all $t$ in $[s, T] v_{\epsilon}(t)=R(s, t) v_{0}^{\epsilon}$. Now we need estimate on $\partial_{x_{i}} v_{\epsilon}$ in order to obtain BV estimate for $v$. Applying Itô's formula to the process $d\left(v_{\epsilon}-v\right)=\left[h\left(v_{\epsilon}\right)-h(v)\right] d W$ and the function $\eta_{\delta}$, taking the integral over $\mathbb{R}^{d}$ and the expectation, we obtain for every $t \in[s, T]$

$$
\begin{aligned}
E \int_{\mathbb{R}^{d}} \eta_{\delta}\left(v_{\epsilon}-v\right)(t) d x= & E \int_{\mathbb{R}^{d}} \eta_{\delta}\left(v_{0}^{\epsilon}-v_{0}\right) d x \\
& +\frac{1}{2} E \int_{\mathbb{R}^{d}} \int_{s}^{t} \eta_{\delta}^{\prime \prime}\left(v_{\epsilon}-v\right)\left[h\left(v_{\epsilon}\right)-h(v)\right]^{2} d \sigma d x .
\end{aligned}
$$


Passing to the limit on $\delta$ to 0 to get for every $t \in[s, T]$

$$
E\left\|\left(v_{\epsilon}-v\right)(t)\right\|_{L^{1}\left(\mathbb{R}^{d}\right)}=E\left\|v_{0}^{\epsilon}-v_{0}\right\|_{L^{1}\left(\mathbb{R}^{d}\right)} .
$$

165 Thus, for every $t \in[s, T], v_{\epsilon}(t) \rightarrow v(t)$ in $L^{1}\left(\Omega \times \mathbb{R}^{d}\right)$.

As P-a.s and for all $t \in[0, T], v_{\epsilon}(t)=v_{\epsilon}(0)+\int_{0}^{t} h\left(v_{\epsilon}\right) d W$ in $W^{1,2}\left(\mathbb{R}^{d}\right)$, using the linear-continuity of the derivation operator $\partial_{x_{i}}: W^{1,2}\left(\mathbb{R}^{d}\right) \rightarrow L^{2}\left(\mathbb{R}^{d}\right)$ for all $i \in\{1, \ldots, d\}$ and the chain-rule derivation formula, we get for all $i \in\{1, \ldots, d\}$ $\partial_{x_{i}} v_{\epsilon}(0)=\partial_{x_{i}} v_{0}^{\epsilon}$ and:

$$
\begin{aligned}
\partial_{x_{i}} v_{\epsilon}(t) & =\partial_{x_{i}} v_{\epsilon}(0)+\partial_{x_{i}} \int_{0}^{t} h\left(v_{\epsilon}\right) d W \\
& =\partial_{x_{i}} v_{\epsilon}(0)+\int_{0}^{t} h^{\prime}\left(v_{\epsilon}\right) \partial_{x_{i}} v_{\epsilon} d W, \text { in } L^{2}\left(\mathbb{R}^{d}\right) .
\end{aligned}
$$

170 Applying Itô's formula with such a process and the function $\eta_{\delta}$ to get that, after taking the integral over $\mathbb{R}^{d}$, the expectation and passing to the limit on $\delta$, for all $t \in[s, T]$

$$
E \int_{\mathbb{R}^{d}}\left|\partial_{x_{i}} v_{\epsilon}\right| d x=E \int_{\mathbb{R}^{d}}\left|\partial_{x_{i}} v_{0}^{\epsilon}\right| d x<\infty
$$

Thus, for all $t \in[s, T]$ and P-a.s, $v_{\epsilon}(t) \in B V\left(\mathbb{R}^{d}\right)$. As for all $t \in[s, T] v_{\epsilon}(t) \rightarrow$ $v(t)$ in $L^{1}\left(\Omega \times \mathbb{R}^{d}\right)$, for a subsequence denoted in the same way, for all $t \in[s, T]$ 175 and P-a.s, $v_{\epsilon}(t) \rightarrow v(t)$ in $L^{1}\left(\mathbb{R}^{d}\right)$. According to Malek-Necas-Otto-RokytaRuzicka [12] p.36, we thus have for all $t \in[s, T]$ and P-a.s

$$
T V_{x}(v(t)) \leqslant \liminf _{\epsilon} T V_{x}\left(v_{\epsilon}(t)\right)
$$


Consequently, taking the expectation thanks to Remark 2 using Fatou's Lemma, (7) then (6), one gets that for every $t \in[s, T]$

$$
E\left[T V_{x}(v(t))\right] \leqslant \liminf _{\epsilon} E\left[T V_{x}\left(v_{\epsilon}(t)\right)\right]=\liminf _{\epsilon} E\left[T V_{x}\left(v_{0}^{\epsilon}\right)\right] \leqslant E\left[T V_{x}\left(v_{0}\right)\right],
$$

and the result holds.

Lemma 3.3. Let $u_{0} \in L^{\infty}\left(\mathbb{R}^{d}\right) \cap B V\left(\mathbb{R}^{d}\right), t>0$, and $u(t)=S(t) u_{0}$. Then:

there exists a constant $C>0$ such that for almost every $t>0$,

$$
\begin{aligned}
\text { i) } & \|u(t)\|_{L^{\infty}\left(\mathbb{R}^{d}\right)} \leqslant\left\|u_{0}\right\|_{L^{\infty}\left(\mathbb{R}^{d}\right)}, \\
\text { ii) } & \int_{\mathbb{R}^{d}}\left|u\left(t_{1}, x\right)-u\left(t_{2}, x\right)\right| d x \leqslant C T V_{x}\left(u_{0}\right)\left|t_{1}-t_{2}\right|, \text { for all } t_{1}, t_{2} \in[0, T], \\
\text { iii) } & T V_{x}(u(t)) \leqslant T V_{x}\left(u_{0}\right) .
\end{aligned}
$$

Proof. These results are classical ones and the proof would be outside the scope of the present work, we refer the reader to Malek-Necas-Otto-Rokyta-Ruzicka [12] p.68.

\subsection{Construction of the approximate solution}

Let us now explain the construction of the approximate solution as introduced in Holden-Risebro [10]. We consider a positive integer $N$, denote by $\Delta=\frac{T}{N}$ and split the time interval by denoting $t_{n}=n \Delta, n \in\{0, \ldots, N\}$ each point of the time discretization. For each step of discretization $\Delta$, we consider the function defined for all $t \in[0, T]$ and $x \in \mathbb{R}^{d}$

$$
u^{\Delta}(t, x)=\left\{\begin{array}{lll}
u^{n}(x) & \text { if } & t=t_{n} \\
R\left(t, t_{n}\right) u^{n}(x) & \text { if } & t \in] t_{n}, t_{n+1}[
\end{array}\right.
$$


where the sequence $\left(u^{n}\right)_{n \in \mathbb{N}}$ is defined by

$$
\begin{cases}u^{0}(x) & =u_{0}(x) \\ u^{n+1}(x) & =S(\Delta) R\left(t_{n+1}, t_{n}\right) u^{n}(x) .\end{cases}
$$

Notations: $\forall n \in\{0, \ldots, N-1\}, t \in[0, T]$ and $x \in \mathbb{R}^{d}$ :

- $u_{-}^{n+1}(x):=R\left(t_{n+1}, t_{n}\right) u^{n}(x)$.

- $\widetilde{u}(t, x):=S\left(t-t_{n}\right) R\left(t_{n+1}, t_{n}\right) u^{n}(x)=S\left(t-t_{n}\right) u_{-}^{n+1}(x)$.

195 Proposition 1. (A priori estimate) There exists a constant $M_{1}$ independent of $n$ and $\Delta$ such that $P$-a.s in $\Omega$ and for all $t \in[0, T]$

$$
\left\|u^{\Delta}(t)\right\|_{L^{\infty}\left(\mathbb{R}^{d}\right)} \leqslant M_{1}:=\max \left(M,\left\|u_{0}\right\|_{L^{\infty}\left(\mathbb{R}^{d}\right)}\right) .
$$

Proof. Let us mention that the construction of $u^{\Delta}$ is done by induction, so the proofs of the associated results also rely on inductive reasoning. Consider $n \in\{0, \ldots, N-1\}$, and $u^{n+1}=S(\Delta) u_{-}^{n+1}$. Thanks to Lemma 3.3 i),

$$
\left\|u^{n+1}\right\|_{L^{\infty}\left(\mathbb{R}^{d}\right)} \leqslant\left\|u_{-}^{n+1}\right\|_{L^{\infty}\left(\mathbb{R}^{d}\right)}, \quad P \text {-a.s. }
$$

Moreover, thanks to Lemma 3.1. P-a.s and $\forall t \in\left[t_{n}, t_{n+1}\right]$

$$
\left\|R\left(t, t_{n}\right) u^{n}\right\|_{L^{\infty}\left(\mathbb{R}^{d}\right)} \leqslant \max \left(M,\left\|u^{n}\right\|_{L^{\infty}\left(\mathbb{R}^{d}\right)}\right)
$$

and particularly for $t=t_{n+1}$, one has P-a.s

$$
\begin{aligned}
\left\|u_{-}^{n+1}\right\|_{L^{\infty}\left(\mathbb{R}^{d}\right)} & =\left\|R\left(t_{n+1}, t_{n}\right) u^{n}\right\|_{L^{\infty}\left(\mathbb{R}^{d}\right)} \\
& \leqslant \max \left(M,\left\|u^{n}\right\|_{L^{\infty}\left(\mathbb{R}^{d}\right)}\right) \\
& \leqslant \max \left(M,\left\|u^{0}\right\|_{L^{\infty}\left(\mathbb{R}^{d}\right)}\right):=M_{1} .
\end{aligned}
$$


Notice that the construction of $u^{\Delta}$ is countable, so $P$-a.s, for all $t \in[0, T]$ and all possible discretization parameter $N \in \mathbb{N}^{*}$ :

$$
\left\|u^{\Delta}(t, .)\right\|_{L^{\infty}\left(\mathbb{R}^{d}\right)} \leqslant M_{1},
$$

where $M_{1}$ does not depend on $\Delta$ and the result holds.

205 Proposition 2. $E\left[T V_{x}\left(u^{i}\right)\right] \leqslant T V_{x}\left(u_{0}\right)$, for every $i \in\{0, \ldots, N\}$.

Proof. Consider $i \in\{0, \ldots, N-1\}$. As $u^{i}=S(\Delta) u_{-}^{i}$, and $u_{-}^{i}=R\left(t_{i}, t_{i-1}\right) u^{i-1}$, using Lemma 3.3 then Lemma 3.2 one gets

$$
E\left[T V_{x}\left(u^{i}\right)\right] \leqslant E\left[T V_{x}\left(u_{-}^{i}\right)\right] \leqslant E\left[T V_{x}\left(u^{i-1}\right)\right]
$$

a reasoning by induction gives us the result.

Lemma 3.4. Let $n \in\{1, \ldots, N\}$ and consider $t \in\left[t_{n}, t_{n+1}[\right.$. Then for any ${ }_{210} \phi \in \mathcal{D}\left(\mathbb{R}^{d}\right)$ :

$$
E \int_{\mathbb{R}^{d}}\left|u^{\Delta}\left(t_{n+1}, x\right)-u^{\Delta}(t, x)\right| \cdot|\phi(x)| d x \leqslant C \Delta T V_{x}\left(u_{0}\right)+\widetilde{C} \sqrt{\Delta},
$$

where $C$ and $\widetilde{C}$ are independent of $\Delta$.

Proof. Let $n \in\{1, \ldots, N\}$ and consider $t \in\left[t_{n}, t_{n+1}\left[\right.\right.$. For all $x \in \mathbb{R}^{d}$,

$$
\begin{aligned}
u^{\Delta}(t, x) & =R\left(t, t_{n}\right) u^{n}(x)=u^{n}(x)+\int_{t_{n}}^{t} h\left(u^{\Delta}(\sigma)\right) d W(\sigma) \\
u^{\Delta}\left(t_{n+1}, x\right) & =u^{n+1}(x) .
\end{aligned}
$$

Thus,

$$
\begin{aligned}
E \int_{\mathbb{R}^{d}}\left|u^{\Delta}\left(t_{n+1}, x\right)-u^{\Delta}(t, x)\right| \cdot|\phi(x)| d x \leqslant & E \int_{\mathbb{R}^{d}}\left|u^{n+1}(x)-u^{n}(x)\right| \cdot|\phi(x)| d x \\
& +E \int_{\mathbb{R}^{d}}\left|\int_{t_{n}}^{t} h\left(u^{\Delta}(\sigma)\right) d W(\sigma)\right| \cdot|\phi(x)| d x
\end{aligned}
$$


Thanks to Lemma 3.3 ii) and Proposition 2 one shows that

$$
\begin{aligned}
& E \int_{\mathbb{R}^{d}}\left|u^{n+1}(x)-u^{n}(x)\right| d x \\
\leqslant & E \int_{\mathbb{R}^{d}}\left|u^{n+1}(x)-u_{-}^{n+1}(x)\right|+\left|u_{-}^{n+1}(x)-u^{n}(x)\right| \cdot|\phi(x)| d x \\
= & E \int_{\mathbb{R}^{d}}\left|S(\Delta) u_{-}^{n+1}(x)-u_{-}^{n+1}(x)\right| \cdot|\phi(x)|+\left|R\left(t_{n+1}, t_{n}\right) u^{n}(x)-u^{n}(x)\right| \cdot|\phi(x)| d x \\
\leqslant & C \Delta \cdot E\left[T V_{x}\left(u_{-}^{n+1}\right)\right]+E \int_{\mathbb{R}^{d}}\left|\int_{t_{n}}^{t_{n+1}} h\left(u^{\Delta}(s, x)\right) d W(s)\right| \cdot|\phi(x)| d x \\
\leqslant & C \Delta \cdot T V_{x}\left(u_{0}\right)+E \int_{\mathbb{R}^{d}}\left|\int_{t_{n}}^{t_{n+1}} h\left(u^{\Delta}(s, x)\right) d W(s)\right| \cdot|\phi(x)| d x .
\end{aligned}
$$

215

Consider $K$ a compact subset of $\mathbb{R}^{d}$ such that $\operatorname{supp} \phi \subset K$ and notice that $\left|t_{n}-t\right| \leqslant \Delta$. Thus by using Cauchy-Schwarz inequality on $\Omega \times K$ and then Itô isometry, one gets

$$
\begin{aligned}
E \int_{\mathbb{R}^{d}}\left|\int_{t_{n}}^{t} h\left(u^{\Delta}(s, x)\right) d W(s)\right| \cdot|\phi(x)| d x & \leqslant\|\phi\|_{L^{\infty}\left(\mathbb{R}^{d}\right)} E \int_{K}\left|\int_{t_{n}}^{t} h\left(u^{\Delta}(s, x)\right) d W(s)\right| d x \\
& \leqslant c\left(E \int_{K}\left|\int_{t_{n}}^{t} h\left(u^{\Delta}(s, x)\right) d W(s)\right|^{2} d x\right)^{\frac{1}{2}} \\
& =c\left(E \int_{K} \int_{t_{n}}^{t} h^{2}\left(u^{\Delta}(s, x)\right) d s d x\right)^{\frac{1}{2}} \\
& \leqslant \widetilde{c} \sqrt{\Delta} .
\end{aligned}
$$

Similarly $E \int_{\mathbb{R}^{d}} \mid \int_{t_{n}}^{t_{n+1}} h\left(u^{\Delta}(s, x) d W(s)|\cdot| \phi(x) \mid d x \leqslant \widetilde{c} \sqrt{\Delta}\right.$, and so $\widetilde{C}=2 \widetilde{c}$.

\subsection{Entropy formulation}

We follow the idea of Peyroutet [14] for introducing the entropy formulation satisfied by the approximate solution. In order to do this, consider

$$
\widetilde{u}(t, x)=S\left(t-t_{n}\right) u_{-}^{n+1}(x), t \in\left[t_{n}, t_{n}+1\right] .
$$


As a weak entropy solution of a conservation law, $\widetilde{u}$ satisfies the following condition, for any $\eta \in \mathcal{E}$ and any $(k, \varphi) \in \mathbb{R} \times \mathcal{D}^{+}\left([0, T] \times \mathbb{R}^{d}\right)$ :

$$
\begin{aligned}
\int_{\mathbb{R}^{d}} \eta\left(\widetilde{u}\left(t_{n}\right)\right. & -k) \varphi\left(t_{n}\right) d x-\int_{\mathbb{R}^{d}} \eta\left(\widetilde{u}\left(t_{n+1}\right)-k\right) \varphi\left(t_{n+1}\right) d x \\
& +\int_{\mathbb{R}^{d}} \int_{t_{n}}^{t_{n+1}} \eta(\widetilde{u}-k) \partial_{t} \varphi+F^{\eta}(\widetilde{u}, k) \nabla \varphi d t d x \geqslant 0 .
\end{aligned}
$$

Consider $(k, \varphi, \eta) \in \mathbb{R} \times \mathcal{D}^{+}\left([0, T] \times \mathbb{R}^{d}\right) \times \mathcal{E}, K$ a compact set of $\mathbb{R}^{d}$ such that

225

$\left[t_{n}, t_{n+1}\right]$ of the stochastic differential equation

$$
\left\{\begin{aligned}
d v & =h(v) d W \\
v\left(t=t_{n}\right) & =u^{n} .
\end{aligned}\right.
$$

Applying the Itô formula to the process $v$ and the regular function $\Psi(t, \lambda)=\eta(\lambda-k)$, one gets $P$-a.s:

$$
\begin{aligned}
\eta\left(v\left(t_{n+1}\right)-k\right)= & \eta\left(v\left(t_{n}\right)-k\right)+\int_{t_{n}}^{t_{n+1}} \eta^{\prime}(v(t)-k) h(v(t)) d W(t) \\
& +\frac{1}{2} \int_{t_{n}}^{t_{n+1}} \eta^{\prime \prime}(v(t)-k) h^{2}(v(t)) d t .
\end{aligned}
$$

Remark that $v(t)=u^{\Delta}(t)$ for all $t \in\left[t_{n}, t_{n+1}\left[\right.\right.$ and $v\left(t_{n+1}\right)=\widetilde{u}\left(t_{n}\right)$, in this way, 230 P-a.s:

$$
\begin{aligned}
& \int_{\mathbb{R}^{d}} \eta\left(\widetilde{u}\left(t_{n}, x\right)-k\right) \varphi\left(t_{n}, x\right) d x-\int_{\mathbb{R}^{d}} \eta\left(u^{\Delta}\left(t_{n}, x\right)-k\right) \varphi\left(t_{n}, x\right) d x \\
= & \int_{\mathbb{R}^{d}} \int_{t_{n}}^{t_{n+1}} \eta^{\prime}\left(u^{\Delta}(t, x)-k\right) h\left(u^{\Delta}(t, x)\right) d W(t) \varphi\left(t_{n}, x\right) d x \\
& +\frac{1}{2} \int_{\mathbb{R}^{d}} \int_{t_{n}}^{t_{n+1}} \eta^{\prime \prime}\left(u^{\Delta}(t, x)-k\right) h^{2}\left(u^{\Delta}(t, x)\right) d t \varphi\left(t_{n}, x\right) d x .
\end{aligned}
$$

Moreover,

$$
\int_{\mathbb{R}^{d}} \eta\left(\widetilde{u}\left(t_{n+1}, x\right)-k\right) \varphi\left(t_{n+1}, x\right) d x=\int_{\mathbb{R}^{d}} \eta\left(u^{\Delta}\left(t_{n+1}, x\right)-k\right) \varphi\left(t_{n+1}, x\right) d x .
$$


Thus one first gets, for any P-measurable set $A$

$$
\begin{aligned}
& E\left(\int_{\mathbb{R}^{d}} \eta\left(u^{\Delta}\left(t_{n}, x\right)-k\right) \varphi\left(t_{n}\right) d x 1_{A}-\int_{\mathbb{R}^{d}} \eta\left(u^{\Delta}\left(t_{n+1}, x\right)-k\right) \varphi\left(t_{n+1}, x\right) d x 1_{A}\right) \\
& +E\left(\int_{\mathbb{R}^{d}} \int_{t_{n}}^{t_{n+1}} \eta^{\prime}\left(u^{\Delta}(t, x)-k\right) h\left(u^{\Delta}(t, x)\right) d W(t) \varphi\left(t_{n}, x\right) d x 1_{A}\right) \\
& +\frac{1}{2} E\left(\int_{\mathbb{R}^{d}} \int_{t_{n}}^{t_{n+1}} \eta^{\prime \prime}\left(u^{\Delta}(t, x)-k\right) h^{2}\left(u^{\Delta}(t, x)\right) d t \varphi\left(t_{n}, x\right) d x 1_{A}\right) \\
& +E\left(\int_{\mathbb{R}^{d}} \int_{t_{n}}^{t_{n+1}} \eta(\widetilde{u}(t, x)-k) \varphi_{t}(t, x)+F^{\eta}(\widetilde{u}(t, x), k) \nabla \varphi(t, x) d t d x 1_{A}\right) \\
\geqslant & 0 .
\end{aligned}
$$

We propose to approximate $E\left(\int_{\mathbb{R}^{d}} \int_{t_{n}}^{t_{n+1}} \eta(\widetilde{u}(t, x)-k) \varphi_{t}(t, x) d t d x 1_{A}\right)$ by $E\left(\int_{\mathbb{R}^{d}} \int_{t_{n}}^{t_{n+1}} \eta\left(u^{n+1}-k\right) \varphi_{t}(t, x) d t d x 1_{A}\right)$ making an error only of order $\Delta^{2}$. In235 deed, thanks to Lemma 3.3 ii) and Proposition 2

$$
\begin{aligned}
& \left|E\left(\int_{\mathbb{R}^{d}} \int_{t_{n}}^{t_{n+1}} \eta(\widetilde{u}(t)-k) \varphi_{t} d t d x-\int_{\mathbb{R}^{d}} \int_{t_{n}}^{t_{n+1}} \eta\left(u^{n+1}-k\right) \varphi_{t} d t d x 1_{A}\right)\right| \\
\leqslant & C E \int_{\mathbb{R}^{d}} \int_{t_{n}}^{t_{n+1}}\left|\eta(\widetilde{u}(t)-k)-\eta\left(u^{n+1}-k\right)\right| \cdot\left|\varphi_{t}\right| d t d x \\
\leqslant & C\left\|\varphi_{t}\right\|_{\infty} E \int_{K} \int_{t_{n}}^{t_{n+1}}\left|\widetilde{u}(t)-u^{n+1}\right| d t d x . \\
\leqslant & C \int_{t_{n}}^{t_{n+1}} E\left[T V_{x}\left(u_{-}^{n+1}\right)\right]\left|t-t_{n+1}\right| d t \\
\leqslant & C \Delta^{2} T V_{x}\left(u_{0}\right) .
\end{aligned}
$$

In the same way, one shows by using the Lipschitz-continuity of $F^{\eta}(., k)$ that $E\left(\int_{\mathbb{R}^{d}} \int_{t_{n}}^{t_{n+1}} F^{\eta}\left(u^{n+1}(x), k\right) \nabla \varphi d t d x 1_{A}\right)$ is an approximation of $E\left(\int_{\mathbb{R}^{d}} \int_{t_{n}}^{t_{n+1}} F^{\eta}(\widetilde{u}(t, x), k) \nabla \varphi(t, x) d t d x 1_{A}\right)$ with an error of order $\Delta^{2}$. 
Finally we obtain by summing over $n$

$$
\begin{aligned}
& E\left(\sum_{n=0}^{N-1} \int_{\mathbb{R}^{d}} \int_{t_{n}}^{t_{n+1}} \eta\left(u^{\Delta}\left(t_{n+1}, x\right)-k\right) \varphi_{t}(t, x) d t d x 1_{A}\right) \\
& +E\left(\sum_{n=0}^{N-1} \int_{\mathbb{R}^{d}} \int_{t_{n}}^{t_{n+1}} F^{\eta}\left(u^{\Delta}\left(t_{n+1}, x\right), k\right) \nabla \varphi(t, x) d t d x 1_{A}\right) \\
& +E\left(\sum_{n=0}^{N-1} \int_{\mathbb{R}^{d}} \int_{t_{n}}^{t_{n+1}} \eta^{\prime}\left(u^{\Delta}(t, x)-k\right) h\left(u^{\Delta}(t, x)\right) d W(t) \varphi\left(t_{n}, x\right) d x 1_{A}\right) \\
& +\frac{1}{2} E\left(\sum_{n=0}^{N-1} \int_{\mathbb{R}^{d}} \int_{t_{n}}^{t_{n+1}} \eta^{\prime \prime}\left(u^{\Delta}(t, x)-k\right) h^{2}\left(u^{\Delta}(t, x)\right) d t \varphi\left(t_{n}, x\right) d x 1_{A}\right) \\
& +\operatorname{mes}(A) \int_{\mathbb{R}^{d}} \eta\left(u_{0}(x)-k\right) \varphi(0, x) d x-E\left(\int_{\mathbb{R}^{d}} \eta\left(u^{\Delta}(T, x)-k\right) \varphi(T, x) d x 1_{A}\right) \\
& \geqslant-\epsilon \Delta, \text { where } \epsilon \Delta \rightarrow 0 \text { when } \Delta \rightarrow 0 .
\end{aligned}
$$

\subsection{Convergence of the approximate solution}

Consider $A$ a $P$-measurable set, $(k, \varphi, \eta) \in \mathbb{R} \times \mathcal{D}^{+}\left([0, T] \times \mathbb{R}^{d}\right) \times \mathcal{E}$ and denote by $K$ a compact set of $\mathbb{R}^{d}$ such that $\operatorname{supp} \varphi(t,.) \subset K$. Our aim is to pass to the limit in:

$$
\begin{aligned}
E\left(\sum_{n=0}^{N-1} \int_{\mathbb{R}^{d}} \int_{t_{n}}^{t_{n+1}} \eta^{\prime}\left(u^{\Delta}(t, x)-k\right) h\left(u^{\Delta}(t, x)\right) d W(t) \varphi\left(t_{n}, x\right) d x 1_{A}\right) & :=I_{1}^{\Delta} \\
+\frac{1}{2} E\left(\sum_{n=0}^{N-1} \int_{\mathbb{R}^{d}} \int_{t_{n}}^{t_{n+1}} \eta^{\prime \prime}\left(u^{\Delta}(t, x)-k\right) h^{2}\left(u^{\Delta}(t, x)\right) d t \varphi\left(t_{n}, x\right) d x 1_{A}\right) & :=I_{2}^{\Delta} \\
+E\left(\sum_{n=0}^{N-1} \int_{\mathbb{R}^{d}} \int_{t_{n}}^{t_{n+1}} \eta\left(u^{\Delta}\left(t_{n+1}, x\right)-k\right) \varphi_{t}(t, x) d t d x 1_{A}\right) & :=I_{3}^{\Delta} \\
+E\left(\sum_{n=0}^{N-1} \int_{\mathbb{R}^{d}} \int_{t_{n}}^{t_{n+1}} F^{\eta}\left(u^{\Delta}\left(t_{n+1}, x\right), k\right) \nabla \varphi(t, x) d t d x 1_{A}\right) & :=I_{4}^{\Delta} \\
+\operatorname{mes}(A) \int_{\mathbb{R}^{d}} \eta\left(u_{0}(x)-k\right) \varphi(0, x) d x & \geqslant-\epsilon \Delta,(8)
\end{aligned}
$$

Due to the random variable, even if strong estimates with respect to variables 
given by the concept of Young measures is appropriate here and the technique is based on the notion of narrow convergence of Young measures (or entropy processes). We refer to Eymard-Gallouët-Herbin [8] and Panov [13]. Since $\left(u^{\Delta}\right)$ is a bounded sequence in $L^{\infty}(Q \times \Omega)$, the associated Young measure sequence 250 $\left(u^{\Delta}\right)$ converges (up to a subsequence still indexed in the same way) to a Young measure denoted $\mathbf{u} \in L^{\infty}(Q \times \Omega \times] 0,1[)$. Furthermore, according to EymardGallouët-Herbin [8], for any Carathéodory function $\Psi$ such that $\Psi\left(., u^{\Delta}\right)$ is uniformly integrable:

$$
E \int_{Q} \Psi\left(u^{\Delta}(t, x)\right) d t d x \rightarrow E \int_{Q} \int_{0}^{1} \Psi(\mathbf{u}(t, x, \alpha)) d \alpha d t d x \text { when } \Delta \rightarrow 0 .
$$

Let us analyze separately terms of 8 .

255

$$
\begin{gathered}
I_{1}^{\Delta} \rightarrow E\left(\int_{\mathbb{R}^{d}} \int_{0}^{T} \int_{0}^{1} \eta^{\prime}(\mathbf{u}(\alpha)-k) h(\mathbf{u}(., \alpha)) d \alpha \varphi d W(t) d x 1_{A}\right):=I_{1} . \\
\left|I_{1}^{\Delta}-I_{1}\right|=\mid E\left(\sum_{n=0}^{N-1} \int_{\mathbb{R}^{d}} \int_{t_{n}}^{t_{n+1}} \eta^{\prime}\left(u^{\Delta}-k\right) h\left(u^{\Delta}\right)\left[\varphi\left(t_{n}\right)-\varphi(t)\right] d W(t) d x 1_{A}\right) \\
+E\left(\int_{\mathbb{R}^{d}} \int_{0}^{T}\left[\eta^{\prime}\left(u^{\Delta}-k\right) h\left(u^{\Delta}\right)-\int_{0}^{1} \eta^{\prime}(\mathbf{u}(., \alpha)-k) h(\mathbf{u}(., \alpha)) d \alpha\right] \varphi(t) d W(t) d x 1_{A}\right) \mid \\
:=\left|I_{1,1}^{\Delta}+I_{1,2}^{\Delta}\right| .
\end{gathered}
$$


Using Cauchy-Schwarz inequality on $\Omega \times K$ and Itô isometry one gets

$$
\begin{aligned}
\left|I_{1,1}^{\Delta}\right| & =\left|E\left(\sum_{n=0}^{N-1} \int_{K} \int_{t_{n}}^{t_{n+1}} \eta^{\prime}\left(u^{\Delta}-k\right) h\left(u^{\Delta}\right)\left[\varphi\left(t_{n}\right)-\varphi(t)\right] d W(t) d x 1_{A}\right)\right| \\
& \leqslant C \sum_{n=0}^{N-1}\left[E \int_{K}\left(\int_{t_{n}}^{t_{n+1}} \eta^{\prime}\left(u^{\Delta}-k\right) h\left(u^{\Delta}\right)\left[\varphi\left(t_{n}\right)-\varphi(t)\right] d W(t)\right)^{2} d x\right]^{\frac{1}{2}} \\
& =C \sum_{n=0}^{N-1}\left[E \int_{K} \int_{t_{n}}^{t_{n+1}}\left[\eta^{\prime}\left(u^{\Delta}-k\right) h\left(u^{\Delta}\right)\left[\varphi\left(t_{n}\right)-\varphi(t)\right]\right]^{2} d t d x\right]^{\frac{1}{2}} \\
& \leqslant C \sum_{n=0}^{N-1}\left[E \int_{t_{n}}^{t_{n+1}} \operatorname{mes}(K) \times \Delta^{2} d t\right]^{\frac{1}{2}} \\
& \leqslant C \sum_{n=0}^{N-1} \Delta^{\frac{3}{2}}=C \sqrt{\Delta} \rightarrow 0
\end{aligned}
$$

Let us show that $I_{1,2}^{\Delta} \rightarrow 0$. Denote $v^{\Delta}=\eta^{\prime}\left(u^{\Delta}-k\right) h\left(u^{\Delta}\right) \varphi$. Thanks to Proposition $11 v^{\Delta}$ is bounded in $L^{2}(Q \times \Omega)$ and there exists $v \in L^{2}(Q \times \Omega)$ such that $v^{\Delta} \rightarrow v$ in the same space. Moreover, $\Psi:(t, x, \omega, \lambda) \mapsto \eta^{\prime}(\lambda-k) h(\lambda) \varphi(t, x)$,

$(t, x, \omega, \lambda) \in Q \times \Omega \times \mathbb{R}$ is a Carathéodory function and $\Psi\left(., u^{\Delta}\right)$ is uniformly integrable as it is bounded in $L^{2}(Q \times \Omega)$. By identification, $v=\int_{0}^{1} \Psi(\mathbf{u}(., \alpha)) d \alpha$. Furthermore, for all $t \in[0, T]$,

$$
\begin{aligned}
I_{t}: L^{2}(Q \times \Omega) & \rightarrow L^{2}\left(\mathbb{R}^{d} \times \Omega\right) \\
\bar{u} & \mapsto \int_{0}^{t} \bar{u}(t, x, \omega) d W(t)
\end{aligned}
$$

is a linear continuous function, and so it is a weakly continuous function from $L^{2}(Q \times \Omega)$ to $L^{2}\left(\mathbb{R}^{d} \times \Omega\right)$. 
265

Consequently, $I_{t}\left(v^{\Delta}\right) \rightarrow I_{t}(v)$ in $L^{2}\left(\mathbb{R}^{d} \times \Omega\right)$. In this manner,

$$
\begin{aligned}
& E\left(\int_{\mathbb{R}^{d}} \int_{0}^{T} \eta^{\prime}\left(u^{\Delta}-k\right) h\left(u^{\Delta}\right) \varphi d W(t) d x 1_{A}\right) \\
\rightarrow & E\left(\int_{\mathbb{R}^{d}} \int_{0}^{T} \int_{0}^{1} \eta^{\prime}(\mathbf{u}(., \alpha)-k) h(\mathbf{u}(., \alpha)) d \alpha \varphi d W(t) d x 1_{A}\right)
\end{aligned}
$$

and $\left|I_{1,2}^{\Delta}\right| \rightarrow 0$.

$$
\begin{aligned}
& I_{2}^{\Delta} \rightarrow \frac{1}{2} E\left(\int_{Q} \int_{0}^{1} \eta^{\prime \prime}(\mathbf{u}(., \alpha)-k) h^{2}(\mathbf{u}(., \alpha)) d \alpha \varphi d t d x 1_{A}\right):=I_{2} . \\
&\left|I_{2}^{\Delta}-I_{2}\right|= \frac{1}{2} \mid E\left(\sum_{n=0}^{N-1} \int_{\mathbb{R}^{d}} \int_{t_{n}}^{t_{n+1}} \eta^{\prime \prime}\left(u^{\Delta}-k\right) h^{2}\left(u^{\Delta}\right)\left[\varphi\left(t_{n}\right)-\varphi(t)\right] d t d x 1_{A}\right) \\
&+E \int_{Q} \eta^{\prime \prime}\left(u^{\Delta}-k\right) h^{2}\left(u^{\Delta}\right) \varphi(t) d t d x 1_{A} \\
&-E \int_{Q} \int_{0}^{1} \eta^{\prime \prime}(\mathbf{u}(., \alpha)-k) h^{2}(\mathbf{u}(., \alpha)) d \alpha \varphi(t) d t d x 1_{A} \mid \\
&:= \frac{1}{2}\left|I_{2,1}^{\Delta}+I_{2,2}^{\Delta}\right| . \\
&\left|I_{2,1}^{\Delta}\right| \leqslant E\left(\sum_{n=0}^{N-1} \int_{K} \int_{t_{n}}^{t_{n+1}}\left|\eta^{\prime \prime}\left(u^{\Delta}-k\right) h^{2}\left(u^{\Delta}\right)\left[\varphi\left(t_{n}\right)-\varphi(t)\right]\right| d t d x\right) \\
& \leqslant C \sum_{n=0}^{N-1} \Delta^{2} \rightarrow 0 .
\end{aligned}
$$

Note that $\Psi(t, x, \omega, \lambda)=\eta^{\prime \prime}(\lambda-k) h^{2}(\lambda) \varphi(t, x) 1_{A}$ is a Carathéodory function 270 such that $\Psi\left(., u^{\Delta}\right)$ is uniformly integrable, thus $I_{2,2}^{\Delta} \rightarrow 0$ and the result holds. Using the same techniques we show the following convergences.

$$
I_{3}^{\Delta} \rightarrow E\left(\int_{Q} \int_{0}^{1} \eta(\mathbf{u}(., \alpha)-k) d \alpha \varphi_{t} d t d x 1_{A}\right):=I_{3} .
$$




$$
I_{4}^{\Delta} \rightarrow E\left(\int_{Q} \int_{0}^{1} F^{\eta}(\mathbf{u}(., \alpha), k) d \alpha \nabla \varphi d t d x 1_{A}\right):=I_{4} .
$$

Finally, for all $(k, \varphi, \eta) \in \mathbb{R} \times \mathcal{D}^{+}\left([0, T] \times \mathbb{R}^{d}\right) \times \mathcal{E}$ and any $P$-measurable set $A$ :

$$
\begin{aligned}
& E\left(\int_{\mathbb{R}^{d}} \int_{0}^{T} \int_{0}^{1} \eta^{\prime}(\mathbf{u}(\alpha)-k) h(\mathbf{u}(., \alpha)) d \alpha \varphi d W(t) d x 1_{A}\right) \\
& +\frac{1}{2} E\left(\int_{Q} \int_{0}^{1} \eta^{\prime \prime}(\mathbf{u}(., \alpha)-k) h^{2}(\mathbf{u}(., \alpha)) d \alpha \varphi d t d x 1_{A}\right) \\
& +E\left(\int_{Q} \int_{0}^{1}\left[\eta(\mathbf{u}(., \alpha)-k) \varphi_{t}+F^{\eta}(\mathbf{u}(., \alpha), k) \nabla \varphi\right] d \alpha d t d x 1_{A}\right) \\
& +\operatorname{mes}(A) \int_{\mathbb{R}^{d}} \eta\left(u_{0}(x)-k\right) \varphi(0, x) d x \geqslant 0 .
\end{aligned}
$$

${ }_{275}$ Hence $\mathbf{u}$ is a measure-valued entropy solution in the sense of Definition 2.2 Thanks to Theorem 2.3, $\mathbf{u}$ is the unique stochastic entropy solution in the sense of Definition 2.1 and we denote it by $u$. Hence, all the sequence of approximate solution $u^{\Delta}$ converges strongly to $u$ in $L_{l o c}^{1}(\Omega \times Q)$. In addition, since $u^{\Delta}$ is bounded in $L^{\infty}(\Omega \times Q)$, all the sequence converges strongly in $L_{l o c}^{p}(\Omega \times Q)$ for any finite $\mathrm{p}$ and the proof of Theorem 1.1 is complete.

\section{Numerical experiments}

We propose here an application of this splitting method to the stochastic burgers' equation in the one-dimensional case:

$$
\left.d u+f(u)_{x} d t=\lambda h(u) d w \text { in } \Omega \times\right] 0,1[\times \mathbb{R},
$$

where $\lambda \in \mathbb{R}$ is a parameter, $f(u)=u^{2}$ and $h: \mathbb{R} \rightarrow \mathbb{R}$ is defined by

$$
h(x)= \begin{cases}(x+1)(1-x) & \text { if }-1<x<1 \\ 0 & \text { else. }\end{cases}
$$


Remark 3. As the flux function $f$ is locally Lipschitz-continuous in $\mathbb{R}$ and as we work with solution explicitly bounded by constant independent of $f$, following Remark 1 we are in the framework presented in the previous section.

The algorithm of discretization is the following one: with an Euler-Maruyama method, we solve the stochastic differential equation (2) and, for solving the conservation law (3), we use a Godunov scheme. We implement simulations with the following initial condition:

$$
u_{0}(x)= \begin{cases}\frac{1}{2} & \text { if } x<0 \\ 0 & \text { else }\end{cases}
$$

To illustrate our proposal, we give a simulation of the solution in the deterministic case (i.e. when $\lambda=0$ ) and for various values of the noise (respectively for $295 \lambda$ equal to $0.1,0.3$ and 0.8$)$. We get the following graphics in the $(x, t)$ plane when $x$ varies between -1 and 1 , with $\Delta_{x}=0.005$ and $\Delta_{t}=\frac{\Delta_{x}}{6}$. 


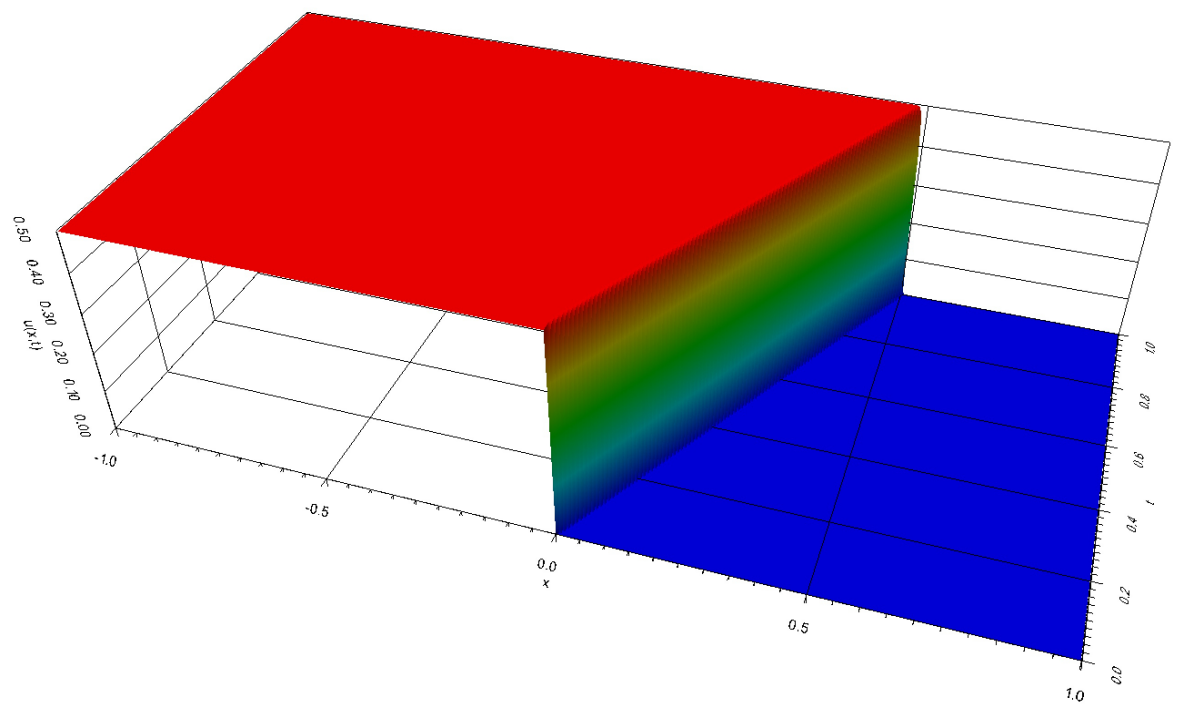

Figure 1: Determinist case : $\lambda=0$

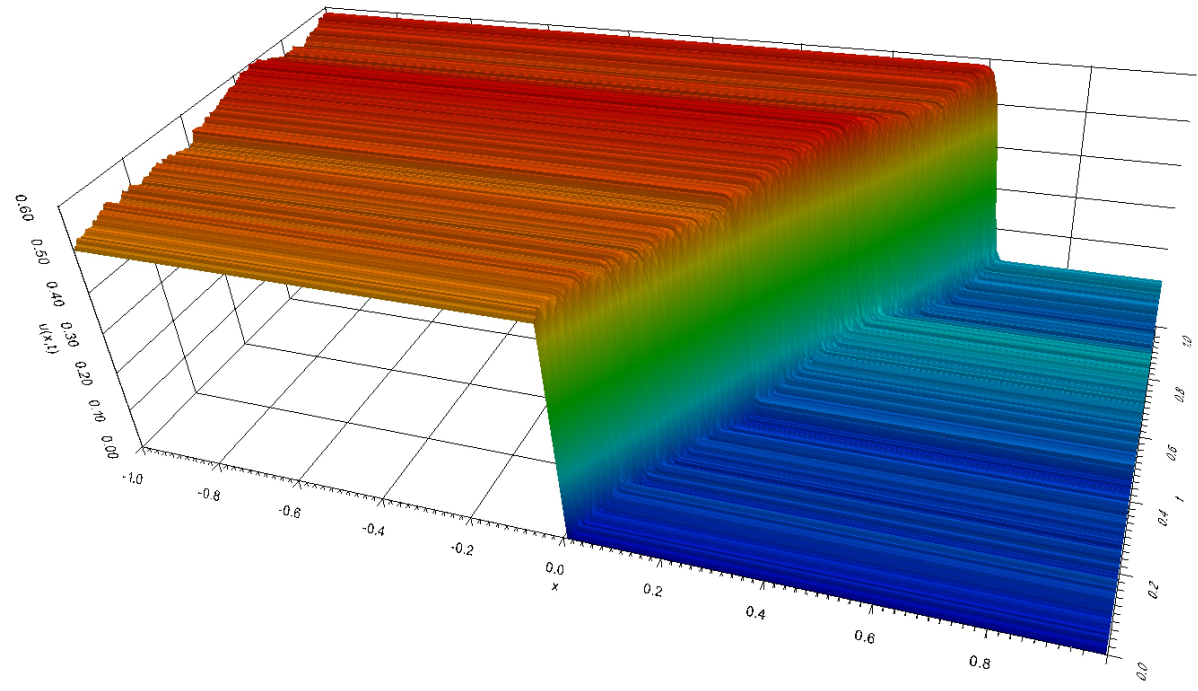

Figure 2: Stochastic case : $\lambda=0.1$ 


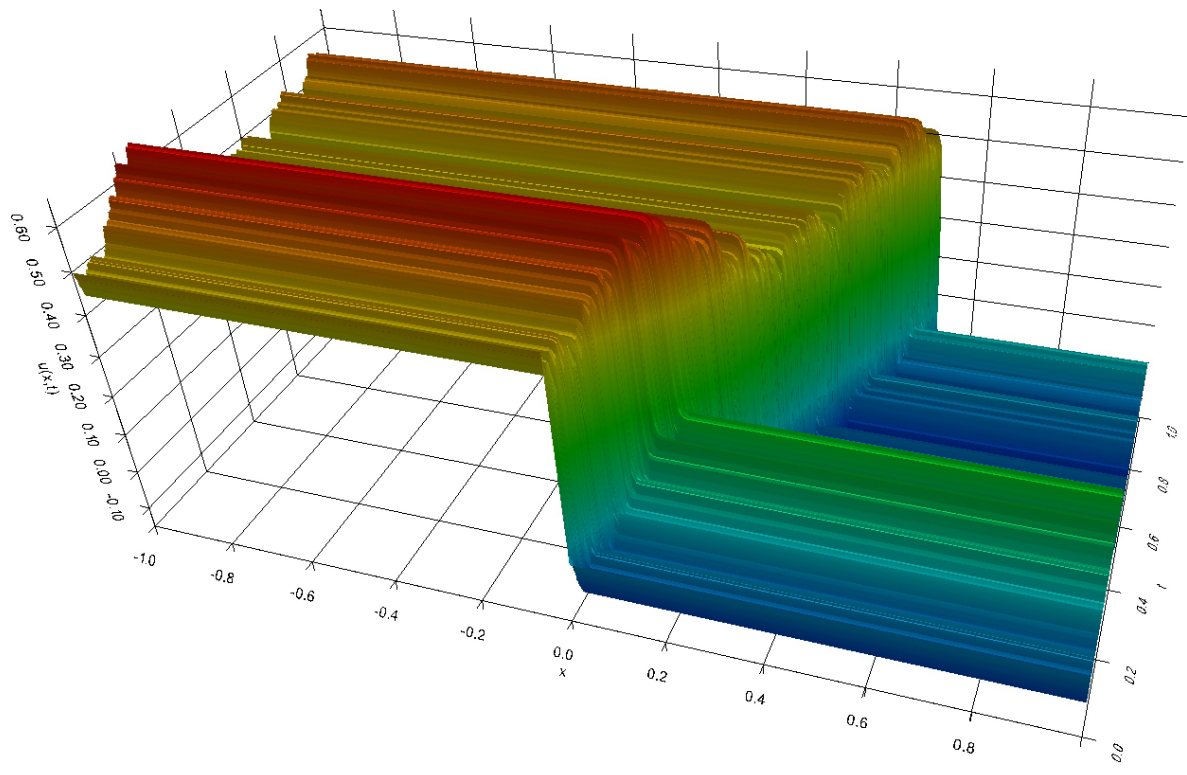

Figure 3: Stochastic case : $\lambda=0.3$

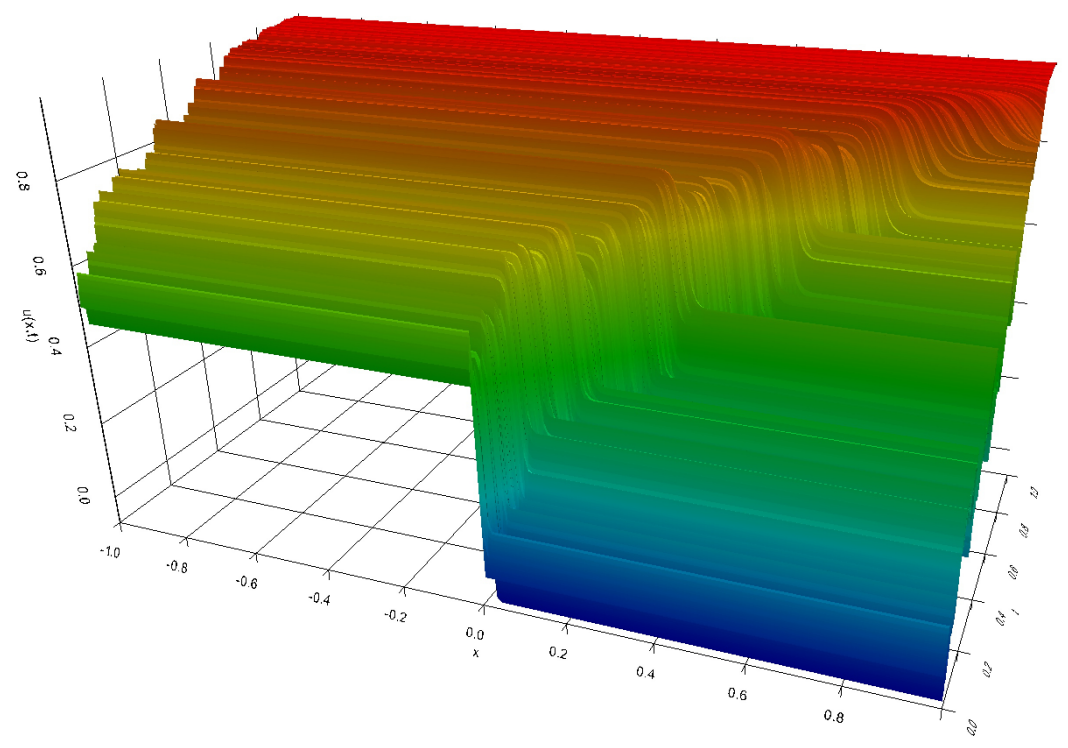

Figure 4: Stochastic case : $\lambda=0.8$ 
Remark 4. Note that we conserve in the stochastic case the propagation of a single choc wave and that the stochastic perturbation seems to act only on the

300 the free software Scilab.

\section{References}

[1] C. Bauzet, On a time-splitting method for a stochastic conservation law with Dirichlet boundary condition and numerical experiments, J. Evol. Eq. 14 (2) (2014) 333-356.

[2] C. Bauzet, G. Vallet, P. Wittbold, The Cauchy problem for a conservation law with a multiplicative stochastic perturbation, J. Hyperbol. Differ. Eq. 9 (4) (2012) 661-709.

[3] C. Bauzet, G. Vallet, P. Wittbold, The Dirichlet problem for a conservation law with a multiplicative stochastic perturbation, J. Funct. Anal. 4 (266) (2014) 2503-2545.

[4] G.-Q. Chen, Q. Ding, K. H. Karlsen, On nonlinear stochastic balance laws, Arch. Ration. Mech. Anal. 204 (3) (2012) 707-743.

URL http://dx .doi . org/10.1007/s00205-011-0489-9 
[5] G. Da Prato, J. Zabczyk, Stochastic equations in infinite dimensions, vol. 44 of Encyclopedia of Mathematics and its Applications, Cambridge University Press, Cambridge, 1992.

URL http://dx.doi.org/10.1017/CB09780511666223

[6] A. Debussche, J. Vovelle, Scalar conservation laws with stochastic forcing, J. Funct. Anal. 259 (4) (2010) 1014-1042.

URL http://dx.doi.org/10.1016/j.jfa.2010.02.016

[7] L. C. Evans, R. F. Gariepy, Measure theory and fine properties of functions, Studies in Advanced Mathematics, CRC Press, Boca Raton, FL, 1992.

[8] R. Eymard, T. Gallouët, R. Herbin, Existence and uniqueness of the entropy solution to a nonlinear hyperbolic equation, Chinese Ann. Math. Ser. B 16 (1) (1995) 1-14.

[9] J. Feng, D. Nualart, Stochastic scalar conservation laws, J. Funct. Anal. 255 (2) (2008) 313-373.

URL http://dx.doi.org/10.1016/j.jfa.2008.02.004

[10] H. Holden, N. H. Risebro, A stochastic approach to conservation laws, Third International Conference on Hyperbolic Problems, Vol. I, II (Uppsala, 1990), Studentlitteratur, Lund, (1991) 575-587. 
[11] I. Kröker, C. Rohde, Finite volume schemes for hyperbolic balance laws with multiplicative noise, Appl. Numer. Math. 62 (4) (2012) 441-456.

[12] J. Málek, J. Necas, M. Rokyta, M. Ruzicka, Weak and measure-valued solutions to evolutionary PDEs, vol. 13 of Applied Mathematics and Mathematical Computation, Chapman \& Hall, London, 1996.

[13] E. Y. Panov, On measure-valued solutions of the Cauchy problem for a first-order quasilinear equation, Izv. Ross. Akad. Nauk Ser. Mat. 60 (2) (1996) 107-148.

URL http://dx.doi .org/10.1070/IM1996v060n02ABEH000073

[14] F. Peyroutet, Etude d'une méthode de splitting pour des lois de conservation scalaires avec terme de source, Ph.D. thesis, U.P.P.A. (1999). 